Pacific Journal of Mathematic 


\section{ON FACETS WITH NON-ARBITRARY SHAPES}

\section{Peter Kleinschmidt}

It is proved that the shape of a facet of a $d$-polytope with $d+3$ vertices can be arbitrarily preassigned. A minimal example of a 4-polytope with 8 vertices which does not have this property is described.

1. Introduction. The shape of a facet $F$ of a polytope $P$ is said to be arbitrarily preassignable if, given any polytope $F^{\prime}$ combinatorially equivalent to $F$ there is a polytope $P^{\prime}$ combinatorially equivalent to $P$ such that $F^{\prime}$ is a facet of $P^{\prime}$ and $F^{\prime}$ is the image of $F$ under the combinatorial isomorphism which maps $P$ onto $P^{\prime}$.

In [3] Barnette and Grünbaum proved that the shape of one $n$ gonal 2 -face $F$ of a 3 -polytope can be any preassigned convex $n$-gon $F^{\prime}$. They ask to what extent their result holds in higher dimensions. They mention that there is an 8-polytope $P$ with 12 vertices such that the shape of one of its 7-dimensional faces can not be arbitrarily chosen, and they conjecture that a similar example can be found already in four dimensions.

In [4], such a 4-polytope with 13 vertices is described. We shall describe a smaller example of this type in the proof of our first theorem:

THEOREM. There is a 4-polytope with 8 vertices such that the shape of one of its 3-faces can not be arbitrarily preassigned.

From the results in [3] and the following lemma we know that the above theorem yields a minimal example of such a polytope.

Lemma. Let $P$ be a d-polytope with $d+3$ vertices. Then the shape of any facet of $P$ can be arbitrarily preassigned.

Proof of the theorem. We shall prove the theorem by describing a 4-polytope $P$, the facets of which are given by their vertices in Table 1.

$P$ possesses 15 facets, 14 of them being tetrahedra and one an octahedron. The vertices of the octahedron are labelled like it is described in Figure 1.

First of all, we have to show that the complex described in Table 1 is isomorphic to the boundary-complex of a convex polytope. Those 3-polytopes given in Table 1 which do not contain the vertex 1 , are either an octahedron (235678) or the convex hull of the vertex 
TABLE 1

\begin{tabular}{ll}
\hline 235678 & 1248 \\
1237 & 2348 \\
1347 & 1568 \\
1467 & 1458 \\
4567 & 1268 \\
1267 & 3458 \\
3457 & 1234 \\
1456 & \\
\hline
\end{tabular}

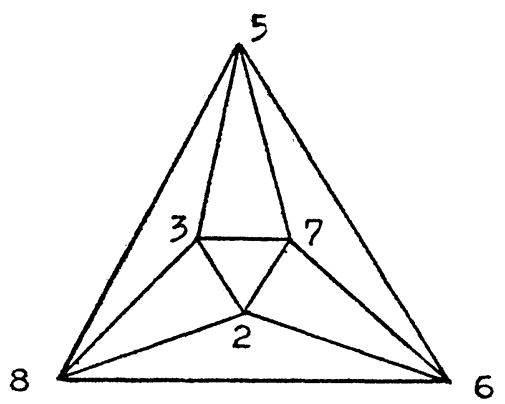

FiguRe 1

4 and a 2 -face of the octahedron $(4567,3457,2348,3458)$. Consequently, the boundary-complex of a pyramid over an octahedron contains a subcomplex isomorphic to the complex formed by these five 3-polytopes. The underlying set of this complex is homeomorphic to a 3-ball.

Using the result of [2], we obtain the following special formulation of Theorem 9 in [5]: If $B$ is a complex formed by 3 -faces in the boundary-complex of a 4-pyramid, and if the underlying set of $B$ is homeomorphic to a 3-ball, then there is a 4-polytope whose set of facets consists of a set isomorphic to $B$ and all 3-polytopes which are the convex hull of a new vertex and the boundary cells of $B$.

Applying this theorem to the complex given in Table 1, we see that it is isomorphic to the boundary-complex of a 4-polytope. We now prove the following:

(1) There is no polytope $P^{\prime}$ combinatorially equivalent to $P$ such that those vertices of $P^{\prime}$ which correspond to the vertices $2,3,5$ and 6 lie in one plane.

We assume that there is a polytope of the type $P^{\prime}$ and regard its Schlegel-diagram $\mathscr{P}^{\prime}$ with basis 1234. Easy calculations show that in $\mathscr{P}^{\prime}$ the vertices $2,3,5$ and 6 are still coplanar (we use the same symbols for vertices of $P^{\prime}$ and their images in $\left.\mathscr{P}^{\prime}\right)$. So we restrict our attention to $\mathscr{P}^{\prime}$ which we assume to lie in a 3 -dimensional space. 


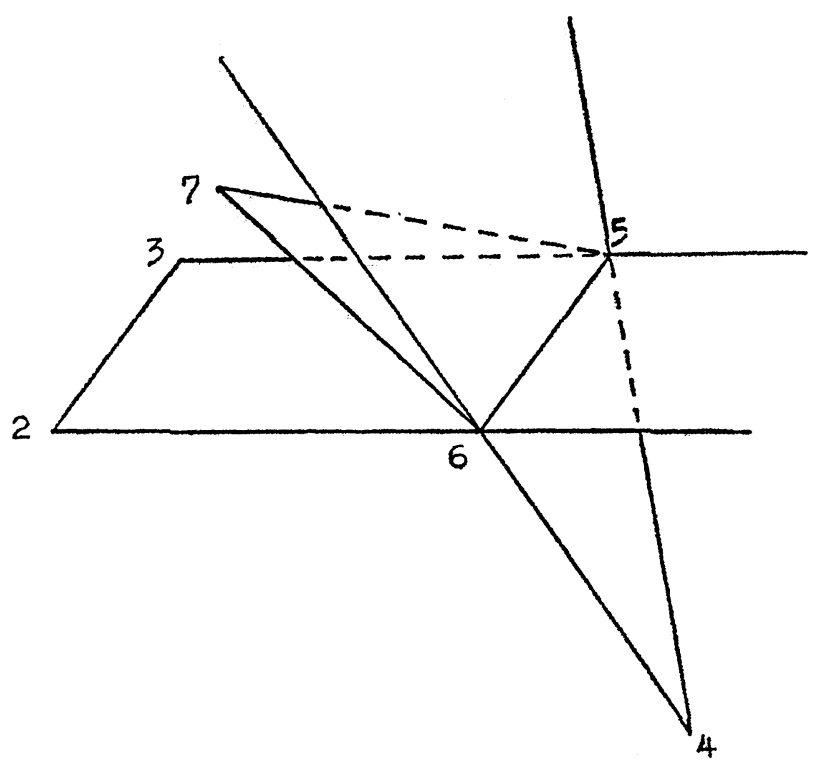

Figure 2

Let $H_{1}$ be the open halfspace which contains the vertex 1 and is bounded by the plane spanned by $2,3,5$ and 6 . Then the vertex 7 lies in $H_{1}$, for otherwise 1237 and 235678 would have common inner points.

Let $\mathrm{H}_{2}$ be the open halfspace which does not contain the vertex 1 and is bounded by the plane spanned by 456. Then 7 lies in $H_{2}$, for otherwise 1456 and 4567 would have common inner points. These arguments yield the following incidences: 7 lies in $H_{1} \cap H_{2}$, the edge 23 lies in $H_{2}$ and 4 does not lie in $H_{1}$.

From this we may conclude that 235678 and 4567 have common inner points (see Figure 2), a contradiction. So we have proved our assumption to be false and consequently, (1) holds. It follows immediately from (1) that an octahedron, the 3 diagonals of which meet in one point, can not be a facet of a polytope combinatorially equivalent to $P$. Thus the theorem is proved.

ReMARKs. A polytope $P$ is said to be projectively unique provided every polytope $P^{\prime}$ combinatorially equivalent to $P$ is projectively equivalent to $P$. The 8 -polytope with 12 vertices mentioned above is a projectively unique one and possesses a facet $F$ which is not projectively unique. Thus in this example, the shape of $F$ may only be arbitrarily chosen within the class of polytopes which are projectively equivalent to $F$.

Our example $P$ reveals another phenomenon concerning the freedom of choice of the shape of a facet: In a polytope combina- 
torially equivalent to $P$, the intersection of the segment 36 and the triangle 257 has to be an inner point of 257. For, if the intersection were in the boundary of 257 , we would have a contradiction to (1). Or, if the intersection were empty, the Schlegel-diagram of $P$ obtained from a projection onto the facet 1234 could be subdivided in such a way that the new diagram would be isomorphic to the nonpolyhedral diagram constructed in [1]. The underlying polyhedron of this diagram, however, can not be 1234 (see [1]), which contradicts our assumption.

Consequently, any metrical type of an octahedron can be preassigned to be a facet of $P$, only if the corresponding vertices of $P$ and the octahedron are labelled in the right way.

It would be interesting to find other phenomena which limit the freedom of preassigning the shape of a facet.

Proof of lemma. Let $P$ be a $d$-polytope with $d+3$ vertices possessing a facet $F$ with $d+2$ vertices. Then $P$ is a pyramid with basis $F$, and any polytope $F^{\prime}$ combinatorially equivalent to $F$ can serve as a basis for a polytope combinatorially equivalent to $P$.

Now let $P$ be a $d$-polytope with $d+3$ vertices possessing a facet $F$ with less than $d+2$ vertices, and let $F^{\prime \prime}$ be any polytope combinatorially equivalent to $F$. Then there is a projective transformation $f$ which is permissible for $F$ and maps $F$ onto $F^{\prime}$. If we extend $f$ in a suitable way to the affine space spanned by $P$, we obtain a projective transformation $g$ which is permissible for $P$ and maps $P$ onto a polytope $P^{\prime} . \quad P^{\prime}$ is of course combinatorially equivalent to $P$ and possesses all the required properties. Taking $k$-fold pyramids over the 4-polytope described in the theorem gives $(k+4)$-polytopes with $k+8$ vertices with $k$-fold pyramids over an octahedron as facets whose shape can not be preassigned. Consequently, the lemma is the best possible.

ACKNowledgment. I wish to thank Branko Grünbaum and the referee for making many suggestions for the improvement of this paper.

\section{REFERENCES}

1. D. Barnette, Diagrams and Schlegel-Diagrams, Combinatorial Structures and Their Applications, Gordon and Breach, New York, (1970), 1-4.

2. — Projections of 3-polytopes, Israel J. Math., 8 (1970), 304-308.

3. D. Barnette and B. Grünbaum, Preassigning the shape of a face, Pacific J. Math., 32 (1970), 299-306.

4. D. Barnette, The triangulations of the 3-sphere with up to 8 vertices, J. Combinatorial Theory, 14 (1973), 37-52. 
5. G. C. Shephard, Sections and projections of convex polytopes, Mathematika, 19 (1972), 144-162.

Received December 30, 1975 and in revised form February 2, 1976.

RUHR-UNIVERSITAET BOCHUM 



\section{PACIFIC JOURNAL OF MATHEMATICS}

\section{EDITORS}

RICHARD ARENS (Managing Editor) University of California

Los Angeles, California 90024

R. A. Beaumont

University of Washington

Seattle, Washington 98105
J. DUGUNDJI

Department of Mathematics University of Southern Californı

Los Angeles, California 90007

D. Gilbarg and J. Milgram

Stanford University

Stanford, California 94305

\section{ASSOCIATE EDITORS}
E. F. BECKENBACH
B. H. NeumanN
F. WOLF
K. YosHIDA

\section{SUPPORTING INSTITUTIONS}

UNIVERSITY OF BRITISH COLUMBIA

CALIFORNIA INSTITUTE OF TECHNOLOGY

UNIVERSITY OF CALIFORNIA

MONTANA STATE UNIVERSITY

UNIVERSITY OF NEVADA

NEW MEXICO STATE UNIVERSITY

OREGON STATE UNIVERSITY

UNIVERSITY OF OREGON

OSAKA UNIVERSITY
UNIVERSITY OF SOUTHERN CALIFORNIA

STANFORD UNIVERSITY

UNIVERSITY OF TOKYO

UNIVERSITY OF UTAH

WASHINGTON STATE UNIVERSITY

UNIVERSITY OF WASHINGTON

* * * *

AMERICAN MATHEMATICAL SOCIETY

NAVAL WEAPONS CENTER 


\section{Pacific Journal of Mathematics \\ Vol. 65, No. $1 \quad$ September, 1976}

David Lee Armacost, Compactly cogenerated LCA groups ............. 1

Sun Man Chang, On continuous image averaging of probability measures ...... 13

J. Chidambaraswamy, Generalized Dedekind $\psi$-functions with respect to a

polynomial. II................................... 19

Freddy Delbaen, The Dunford-Pettis property for certain uniform algebras ..... 29

Robert Benjamin Feinberg, Faithful distributive modules over incidence

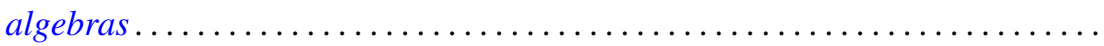

Paul Froeschl, Chained rings . . . . . . . . . . . . . . . . . . . .

John Brady Garnett and Anthony G. O'Farrell, Sobolev approximation by a sum

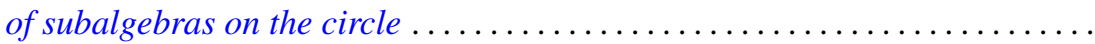

Hugh M. Hilden, José M. Montesinos and Thomas Lusk Thickstun, Closed

oriented 3-manifolds as 3-fold branched coverings of $S^{3}$ of special type.....

Atsushi Inoue, On a class of unbounded operator algebras ................

Peter Kleinschmidt, On facets with non-arbitrary shapes.

Narendrakumar Ramanlal Ladhawala, Absolute summability of Walsh-Fourier

series

Howard Wilson Lambert, Links which are unknottable by maps . . . . . . . . . . .

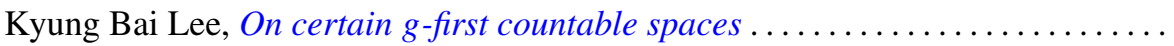

Richard Ira Loebl, A Hahn decomposition for linear maps .................

Moshe Marcus and Victor Julius Mizel, A characterization of functionals on $W_{1}^{p}$ possessing autonomous kernels. I . .

James Miller, Subordinating factor sequences and convex functions of several

variables.

Keith Pierce, Amalgamated sums of abelian l-groups ...

Jonathan Rosenberg, The $C^{*}$-algebras of some real and $p$-adic solvable

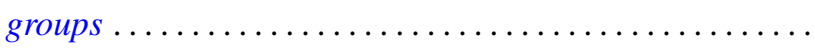

Hugo Rossi and Michele Vergne, Group representations on Hilbert spaces defined

in terms of $\partial_{b}$-cohomology on the Silov boundary of a Siegel domain . .

Mary Elizabeth Schaps, Nonsingular deformations of a determinantal

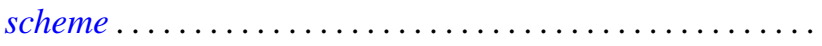

S. R. Singh, Some convergence properties of the Bubnov-Galerkin method...

Peggy Strait, Level crossing probabilities for a multi-parameter Brownian

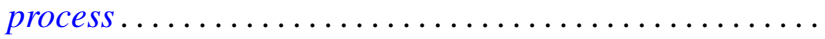

Robert M. Tardiff, Topologies for probabilistic metric spaces .

Benjamin Baxter Wells, Jr., Rearrangements of functions on the ring of integers of

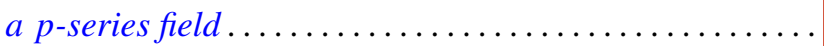

Robert Francis Wheeler, Well-behaved and totally bounded approximate identities for $C_{0}(X)$.

Delores Arletta Williams, Gauss sums and integral quadratic forms over local

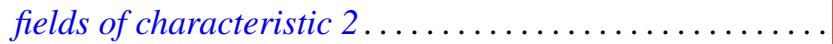

John Yuan, On the construction of one-parameter semigroups in topological 